\title{
Re-evaluation of fatty acid metabolism-related gene expression in nonalcoholic fatty liver disease
}

\author{
MOTOYUKI KOHJIMA ${ }^{1}$, MUNECHIKA ENJOJI ${ }^{1}$, NOBITO HIGUCHI ${ }^{1}$, MASAKI KATO $^{1}$, KAZUHIRO KOTOH $^{1}$, \\ TSUYOSHI YOSHIMOTO ${ }^{2}$, TATSUYA FUJINO ${ }^{2}$, MASAYOSHI YADA ${ }^{1}$, RYOKO YADA ${ }^{2}$, \\ NAOHIKO HARADA ${ }^{2}$, RYOICHI TAKAYANAGI ${ }^{1}$ and MAKOTO NAKAMUTA ${ }^{2}$ \\ ${ }^{1}$ Department of Medicine and Bioregulatory Science, Graduate School of Medical Sciences, Kyushu University, Fukuoka; \\ ${ }^{2}$ Department of Gastroenterology, Kyushu Medical Center, National Hospital Organization, Fukuoka, Japan
}

Received April 18,2007; Accepted May 30, 2007

\begin{abstract}
Nonalcoholic fatty liver disease (NAFLD) is one of the most frequent causes of abnormal liver dysfunction, and its prevalence has markedly increased. We previously evaluated the expression of fatty acid metabolism-related genes in NAFLD and reported changes in expression that could contribute to increased fatty acid synthesis. In the present study, we evaluated the expression of additional fatty acid metabolism-related genes in larger groups of NAFLD $(n=26)$ and normal liver $(n=10)$ samples. The target genes for real-time PCR analysis were as follows: acetyl-CoA carboxylase (ACC) 1, ACC2, fatty acid synthase (FAS), sterol regulatory element-binding protein 1c (SREBP-1c), and adipose differentiation-related protein (ADRP) for evaluation of de novo synthesis and uptake of fatty acids; carnitine palmitoyltransferase 1a (CPT1a), long-chain acyl-CoA dehydrogenase (LCAD), long-chain L-3-hydroxyacylcoenzyme A dehydrogenase $\alpha(\mathrm{HADH} \alpha)$, uncoupling protein 2 (UCP2), straight-chain acyl-CoA oxidase (ACOX),
\end{abstract}

Correspondence to: Dr Munechika Enjoji, Department of Medicine and Bioregulatory Science, Graduate School of Medical Sciences, Kyushu University, 3-1-1 Maidashi, Higashi-ku, Fukuoka 812-8582, Japan

E-mail: enjoji@intmed3.med.kyushu-u.ac.jp

Abbreviations: NAFLD, nonalcoholic fatty liver disease; NASH, nonalcoholic steatohepatitis; ACC, acetyl-CoA carboxylase; FAS, fatty acid synthase, SREBP-1c, sterol regulatory element-binding protein 1c; ADRP, adipose differentiation-related protein; CPT1a, carnitine palmitoyltransferase 1a; LCAD, long-chain acyl-CoA dehydrogenase; HADH, long-chain L-3-hydroxyacyl-coenzyme A dehydrogenase; UCP2, uncoupling protein 2; ACOX, straight-chain acyl-CoA oxidase; BOX, branched-chain acyl-CoA oxidase; CYP, cytochrome P-450; PPAR, peroxisome proliferator-activated receptor; DGAT1, diacylglycerol O-acyltransferase 1; HSL, hormone-sensitive lipase; SOD, superoxide dismutase; GSS, glutathione synthetase; ROS, reactive oxygen species; AMPK, AMP-activated protein kinase

Key words: nonalcoholic fatty liver disease, fatty acid, oxidation, reactive oxygen species branched-chain acyl-CoA oxidase (BOX), cytochrome P450 2E1 (CYP2E1), CYP4A11, and peroxisome proliferatoractivated receptor (PPAR) $\alpha$ for oxidation in the mitochondria, peroxisomes and microsomes; superoxide dismutase (SOD), catalase, and glutathione synthetase (GSS) for antioxidant pathways; and diacylglycerol O-acyltransferase 1 (DGAT1), PPAR $\gamma$, and hormone-sensitive lipase (HSL) for triglyceride synthesis and catalysis. In NAFLD, although fatty acids accumulated in hepatocytes, their de novo synthesis and uptake were up-regulated in association with increased expression of ACC1, FAS, SREBP-1c, and ADRP. Fatty acid oxidation-related genes, LCAD, HADH $\alpha$, UCP2, ACOX, BOX, CYP2E1, and CYP4A11, were all overexpressed, indicating that oxidation was enhanced in NAFLD, whereas the expression of CTP $1 \mathrm{a}$ and PPAR $\alpha$ was decreased. Furthermore, SOD and catalase were also overexpressed, indicating that antioxidant pathways are activated to neutralize reactive oxygen species (ROS), which are overproduced during oxidative processes. The expression of DGAT1 was up-regulated without increased PPAR $\gamma$ expression, whereas the expression of HSL was decreased. Our data indicated the following regarding NAFLD: i) increased de novo synthesis and uptake of fatty acids lead to further fatty acid accumulation in hepatocytes; ii) mitochondrial fatty acid oxidation is decreased or fully activated; iii) in order to complement the function of mitochondria ( $\beta$-oxidation), peroxisomal ( $\beta$-oxidation) and microsomal ( $\omega$-oxidation) oxidation is up-regulated to decrease fatty acid accumulation; iv) antioxidant pathways including SOD and catalase are enhanced to neutralize ROS overproduced during mitochondrial, peroxisomal, and microsomal oxidation; and v) lipid droplet formation is enhanced due to increased DGAT expression and decreased HSL expression. Further studies will be needed to clarify how fatty acid synthesis is increased by SREBP-1c, which is under the control of insulin and AMP-activated protein kinase.

\section{Introduction}

Nonalcoholic fatty liver disease (NAFLD), which is characterized by triglyceride accumulation in hepatocytes (hepatic steatosis), is one of the most frequent causes of 

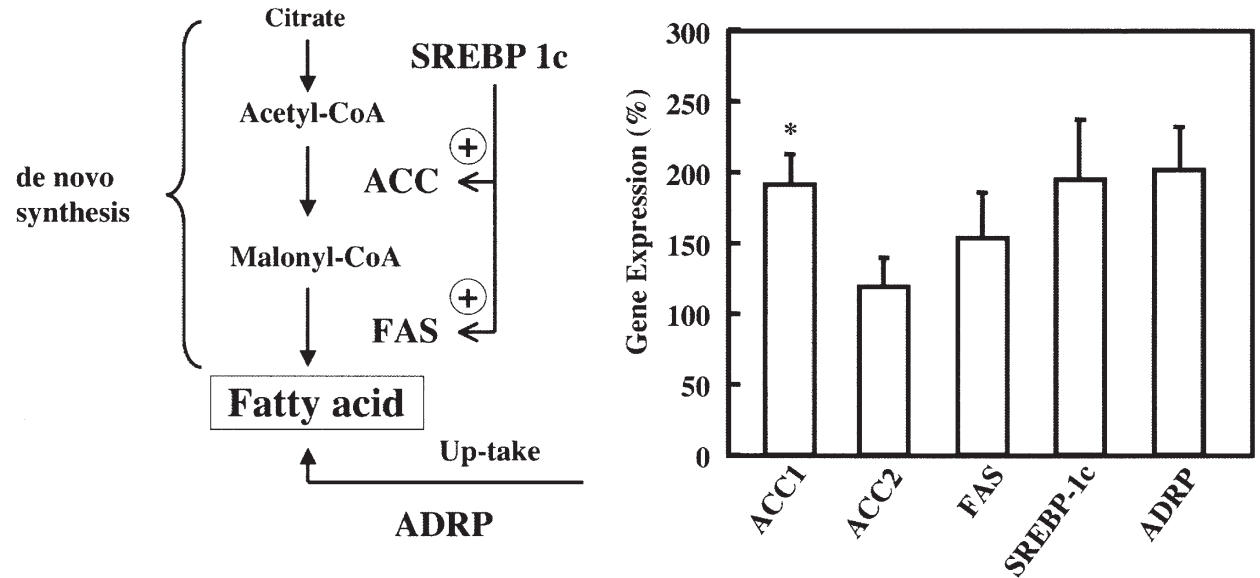

Figure 1. Real-time RT-PCR analysis for gene expression of de novo synthesis and uptake of fatty acids in NAFLD. *p<0.05, a statistically significant difference as compared with the normal liver. ACC, acetyl-CoA carboxylase; FAS, fatty acid synthase; SREBP-1c, sterol regulatory element-binding protein 1c; ADRP, Adipose differentiation-related protein (adipophilin).

abnormal liver function (1-3). The prevalence of NAFLD in the general population is estimated to be between 14 and $24 \%$ (4-6), and it has markedly increased in all segments of the population including children. Furthermore, nonalcoholic steatohepatitis (NASH), a severe form of NAFLD which is accompanied by hepatitis and fibrosis (7), can progress to cirrhosis and hepatic failure (8). It has been reported that $>20 \%$ of patients with NASH develop cirrhosis, half of which subsequently die of liver failure (8). NAFLD is often associated with obesity and/or insulin resistance; however, the precise cause of NAFLD remains unclear. It is important, therefore, to characterize lipid metabolism, particularly fatty acid metabolism, in NAFLD.

Fatty acids in the liver are derived from de novo synthesis and plasma-free fatty acids. Acetyl-CoA is an essential substrate for de novo synthesis and is ultimately converted to stearic acid (C18:0), which also can be desaturated to oleic acid in hepatocytes. Plasma fatty acids are also actively taken up by a specific transporter. Up-regulation of synthesis and/or uptake can result in fatty acid accumulation. Fatty acids in hepatocytes are metabolized by either of 2 pathways; oxidation to generate ATP (e.g. B-oxidation in the mitochondria) or esterification to produce triglycerides, which are either incorporated into lipoproteins for export or stored as lipid droplets within the hepatocytes. Defects in one or both of these pathways can lead to hepatic steatosis.

We previously evaluated the expression of genes related to fatty acid metabolism and reported that de novo synthesis of fatty acids was up-regulated in spite of their accumulation in hepatocytes of patients with NAFLD (9). In this study, using more samples from NAFLD and control livers, we further investigated fatty acid metabolism in NAFLD by re-evaluating the expression of genes involved in de novo synthesis, uptake, oxidation, antioxidant pathways, and triglyceride synthesis and catalysis.

\section{Patients and methods}

Tissue samples were obtained by liver biopsy from 26 patients with histologically diagnosed NAFLD, including 4 patients with NASH, who were admitted to the Kyushu
University Hospital between 2004 and 2006. As a control, normal liver was also obtained by biopsy from 10 men whose liver function tests and histological findings were completely normal. Real-time RT-PCR was performed as previously reported (9). Total RNA was prepared with Trizol reagent (Invitrogen, Carlsbad, CA, USA), and cDNA was synthesized from $1.0 \mu \mathrm{g}$ RNA with GeneAmp ${ }^{\mathrm{TM}}$ RNA PCR (Applied Biosystems, Branchburg, NJ, USA) using random hexamers. Real-time RT-PCR was performed using LightCyclerFastStart DNA Master SYBR-Green 1 (Roche, Tokyo, Japan), according to the manufacturer's instructions. The reaction mixture $(20 \mu \mathrm{l})$ contained LightCycler-FastStart DNA Master SYBR-Green 1, $4 \mathrm{mM} \mathrm{MgCl}_{2}, 0.5 \mu \mathrm{M}$ of the upstream and downstream PCR primers, and $2 \mathrm{ml}$ of the first-strand cDNA as a template. The target genes and their primers are shown in Table I. To control for variations in the reactions, all PCRs were normalized against $\beta$-actin expression. All results are shown as the mean \pm SEM. Comparisons were made by the Mann-Whitney U test.

\section{Results}

Expression of genes related to de novo synthesis and uptake of fatty acids. In the process of fatty acid synthesis, acetylCoA carboxylase (ACC) converts acetyl-CoA, an essential substrate of fatty acids, to malonyl-CoA. Fatty acid synthase (FAS) then utilizes both acetyl-CoA and malonyl-CoA to form palmitic acid (C16:0). In NAFLD, the expression of ACC1 and FAS was 2-fold and 1.5-fold higher, respectively, than that in the normal liver (Fig. 1). Both ACC and FAS are positively regulated by a transcriptional factor, sterol regulatory element-binding protein 1c (SREBP-1c) (1). In NAFLD, SREBP-1c expression was also higher than that in the normal liver (Fig. 1). In addition to de novo synthesis, fatty acids in hepatocytes are transferred from serum by adipose differentiation-related protein (ADRP, adipophilin) (10). ADRP expression in NAFLD was 2-fold higher than in the normal liver (Fig. 1).

Gene expression related to $\beta$-oxidation in mitochondria. Carnitine palmitoyltransferase 1a (CPT1a) is a regulatory 
Table I. Primers used for analysis for expression of fatty acid metabolism-related genes.

\begin{tabular}{|c|c|c|}
\hline Gene & $\begin{array}{l}\text { Forward primer } \\
\text { Reverse primer }\end{array}$ & GenBank $^{\mathrm{TM}}$ accession no. \\
\hline $\mathrm{ACC} 1$ & $\begin{array}{l}\text { GAGGGCTAGGTCTTTCTGGAAG } \\
\text { CCACAGTGAAATCTCGTTGAGA }\end{array}$ & NM-198834 \\
\hline ACC2 & $\begin{array}{l}\text { GCCAGAAGCCCCCAAGAAAC } \\
\text { CGACATGCTCGGCCTCATAG }\end{array}$ & NM-001093 \\
\hline FAS & $\begin{array}{l}\text { AGCTGCCAGAGTCGGAGAAC } \\
\text { TGTAGCCCACGAGTGTCTCG }\end{array}$ & NM-004104 \\
\hline SREBP-lc & $\begin{array}{l}\text { GCGGAGCCATGGATTGCAC } \\
\text { CTCTTCCTTGATACCAGGCCC }\end{array}$ & NM-004176 \\
\hline ADRP & $\begin{array}{l}\text { GGGATCCCTGTCTACCAAGC } \\
\text { AGATGTCGCCTGCCATCACC }\end{array}$ & NM-001122 \\
\hline CPTIa & $\begin{array}{l}\text { TGAGCGACTGGTGGGAGGAG } \\
\text { GAGCCAGACCTTGAAGTAGCG }\end{array}$ & NM-001876 \\
\hline LCAD & $\begin{array}{l}\text { GGTGTTCATCAGTAATGGGTCAT } \\
\text { CACTGTCTGTAGGTGAGCAACTG }\end{array}$ & NM-001608 \\
\hline $\mathrm{HADH} \alpha$ & $\begin{array}{l}\text { GCTAGACCGAGGACAGCAAC } \\
\text { CCTGCTTGAGACCAACTGCT }\end{array}$ & NM-000182 \\
\hline UCP2 & $\begin{array}{l}\text { CACCAAGGGCTCTGAGCATG } \\
\text { TCTACAGGGGAGGCGATGAC }\end{array}$ & NM-003355 \\
\hline ACOX & $\begin{array}{l}\text { TCCTGCCCACCTTGCTICAC } \\
\text { TTGGGGCCGATGTCACCAAC }\end{array}$ & NM-004035 \\
\hline $\mathrm{BOX}$ & $\begin{array}{l}\text { GGGCATTCCACATCCGGTTG } \\
\text { TGGCTCCTGAGCAGATCAGC }\end{array}$ & NM-003500 \\
\hline CYP2E1 & $\begin{array}{l}\text { ATGTCTGCCCTCGGAGTGA } \\
\text { GATGTCCTTCCAGGTAGGTCC }\end{array}$ & NM-000773 \\
\hline CYP4A11 & $\begin{array}{l}\text { AGGAGCTCCAACAGGACCAG } \\
\text { CCTGATGGCTGAAGGCACAC }\end{array}$ & NM-000778 \\
\hline $\operatorname{PPAR} \alpha$ & $\begin{array}{l}\text { CCAGTATTTAGGAAGCTGTCCTG } \\
\text { CGTTGTGTGACATCCCGACAG }\end{array}$ & NM-005036 \\
\hline SOD & $\begin{array}{l}\text { AGGCCGTGTGCGTGCTGAAG } \\
\text { CACCTTTGCCCAAGTCATCTGC }\end{array}$ & NM-000454 \\
\hline Catalase & $\begin{array}{l}\text { CCTTTCTGTTGAAGATGCGGCG } \\
\text { GGCGGTGAGTGTCAGGATAG }\end{array}$ & NM-001752 \\
\hline GSS & $\begin{array}{l}\text { AGAACGCTGCCTTCCTGGAG } \\
\text { CAGTAGCACCAGAGCATTGGG }\end{array}$ & NM-000178 \\
\hline DGAT1 & $\begin{array}{l}\text { GGCATCCTGAACTGGTGTGTG } \\
\text { GAGCTTGAGGAAGAGGATGGTG }\end{array}$ & NM-012079 \\
\hline $\operatorname{PPAR} \gamma$ & $\begin{array}{l}\text { GAACAGATCCAGTGGTTGCAG } \\
\text { GGCATTATGAGACATCCCCAC }\end{array}$ & NM-138712 \\
\hline HSL & $\begin{array}{l}\text { TACCGCAGCCTAGTGCACAC } \\
\text { AGATGGTCTGCAGGAATGGC }\end{array}$ & NM-005357 \\
\hline B-actin & $\begin{array}{l}\text { GCAAGAGAGGCATCCTCACC } \\
\text { CGTAGATGGGCACAGTGTGG }\end{array}$ & NM-001101 \\
\hline
\end{tabular}

enzyme in mitochondria that transfers fatty acids from the cytosol to mitochondria prior to ß-oxidation. ß-oxidation is then catalyzed by enzymes such as long-chain acyl-CoA dehydrogenase (LCAD) and long-chain L-3-hydroxyacylcoenzyme A dehydrogenase $\alpha(\mathrm{HADH} \alpha)$. In NAFLD, CPT1a expression was decreased by $50 \%$, and expression of LCAD and $\mathrm{HADH} \alpha$ was significantly increased 6-fold and 3-fold, respectively, compared with that in the normal liver (Fig. 2). Uncoupling protein 2 (UCP2), a mitochondrial innermembrane protein is emerging as a potential regulator of mitochondrial reactive oxygen species (ROS) production (11). It mediates a proton leak across the inner membrane 

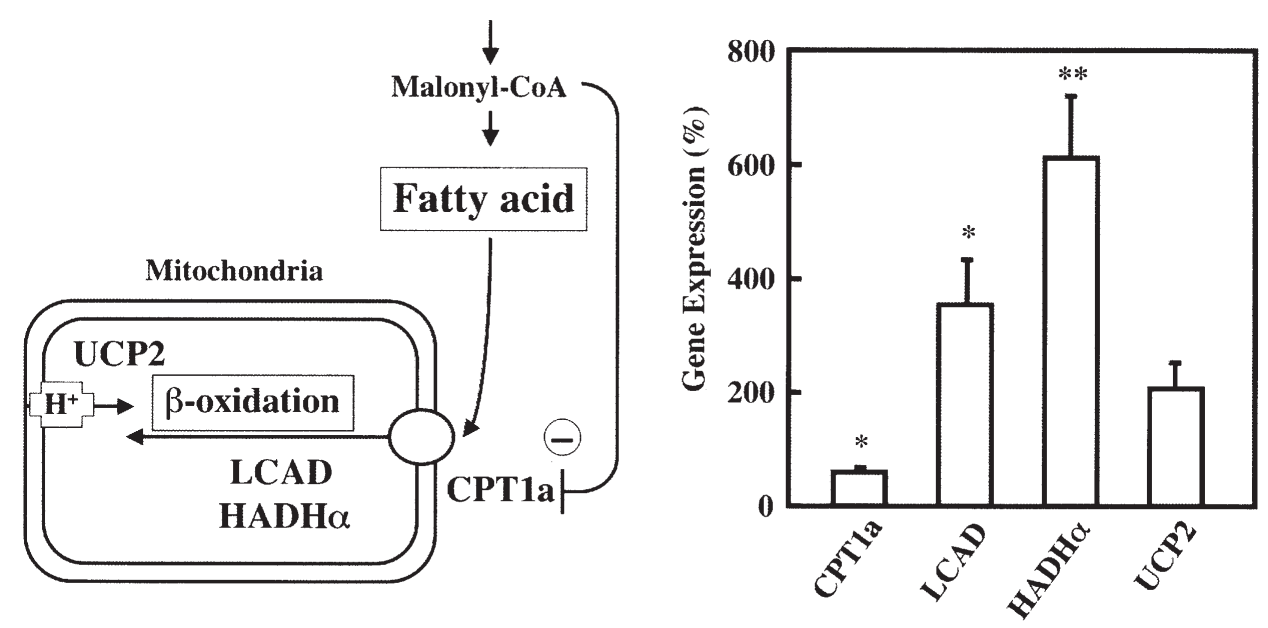

Figure 2. Real time RT-PCR analysis for gene expression of mitochondrial $\beta$-oxidation in NAFLD. ${ }^{*} \mathrm{p}<0.05$ and ${ }^{* *} \mathrm{p}<0.01$ indicate statistically significant differences as compared with the normal liver. CPT1a, carnitine palmitoyltransferase 1a; LCAD, long-chain acyl-CoA dehydrogenase; HADH $\alpha$, long-chain L-3-hydroxyacyl-coenzyme A dehydrogenase $\alpha$; UCP2, uncoupling protein 2.

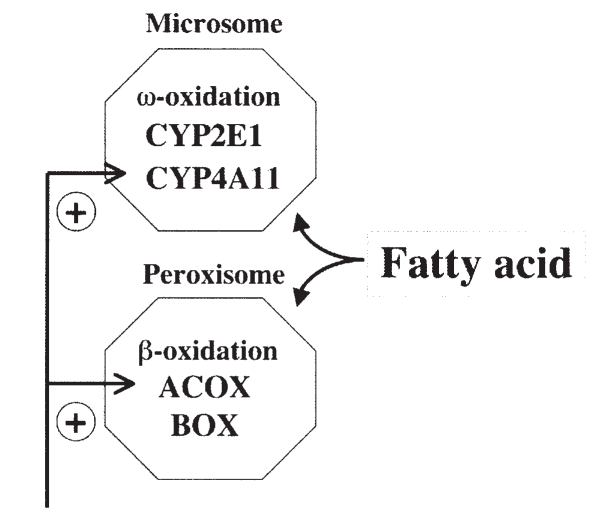

PPAR- $\alpha$

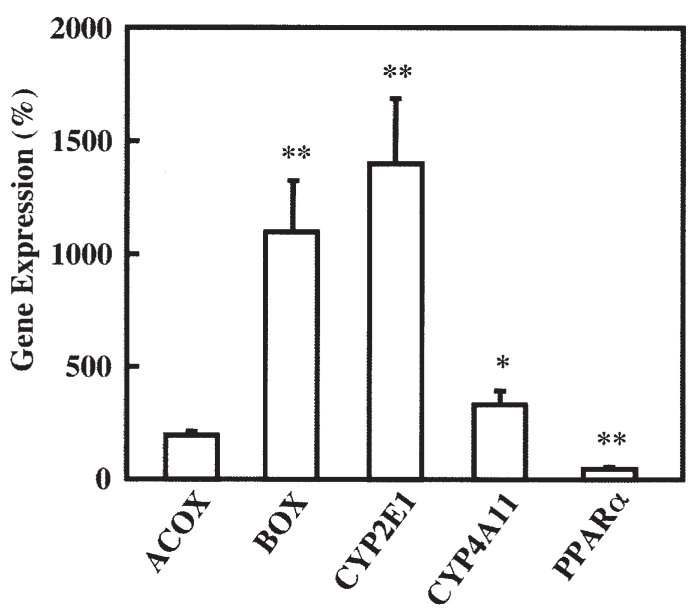

Figure 3. Real time RT-PCR analysis for gene expression of peroxisomal or microsomal oxidation in NAFLD. "p $<0.05$ and ${ }^{* *}$ p $<0.01$ indicate statistically significant differences as compared with the normal liver. ACOX, straight-chain acyl-CoA oxidase; BOX, branched-chain acyl-CoA oxidase; CYP, cytochrome P450; PPAR $\alpha$, peroxisome proliferator-activated receptor $\alpha$.

and uncouples fuel oxidation from adenosine triphosphate (ATP) synthesis (12). UCP2 expression in NAFLD was 2-fold higher than in the normal liver.

Expression of other genes related to fatty acid oxidation. When cytosolic fatty acids accumulate due to impairment of oxidative capacity in mitochondria, alternative pathways in the peroxisomes ( $\beta$-oxidation) and in microsomes $(\omega$ oxidation) are activated. In peroxisomal $\beta$-oxidation, straightchain acyl-CoA oxidase (ACOX) and branched-chain acylCoA oxidase (BOX) are responsible for the initial oxidation of very-long-chain fatty acyl-CoAs. In NAFLD, the expression of ACOX and BOX was increased 2-fold and 10-fold, respectively, compared with that in the normal liver (Fig. 3). In microsomal w-oxidation, CYP2E1 and CYP4A11, which are inducible hepatic microsomal cytochrome P-450s, can initiate the autopropagative process of lipid oxidation. In NAFLD, the expression of CYP2E1 and CYP4A11 was significantly higher (14-fold and 4-fold, respectively) than in the normal liver (Fig. 3). Peroxisome proliferator-activated receptor (PPAR) $\alpha$, a transcriptional factor, up-regulates the expression of a suite of genes that includes peroxisomal and mitochondrial $\beta$-oxidation enzymes as well as CYP4A. In NAFLD, PPAR $\alpha$ expression was significantly decreased by $50 \%$ compared with that in the normal liver (Fig. 3).

Expression of genes related to antioxidant pathways. ROS are formed during the process of fatty acid oxidation. They are eliminated by antioxidant enzymes such as superoxide dismutase (SOD) and catalase, and by compounds such as glutathione, which is produced by glutathione synthetase (GSS). In NAFLD, the expression of SOD and catalase was increased 5-fold and 10-fold, respectively, compared with that in normal liver, whereas GSS expression was unchanged (Fig. 4).

Expression of genes related to lipid droplet formation. Fatty acids are also metabolized by esterification to produce 

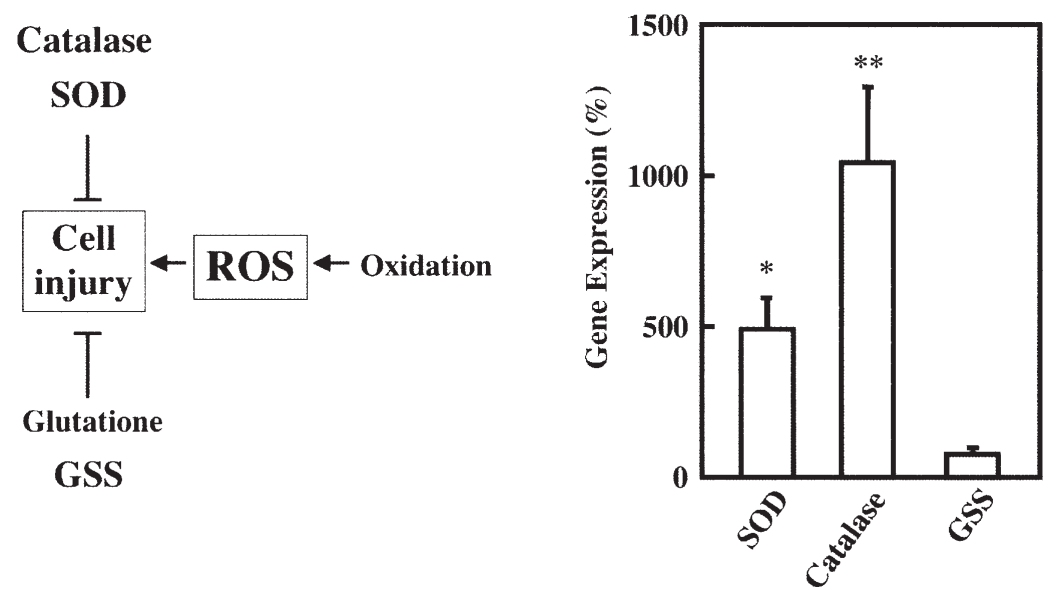

Figure 4. Real time RT-PCR analysis for expression of antioxidation-related genes in NAFLD. ${ }^{*} \mathrm{p}<0.05$ and ${ }^{* *} \mathrm{p}<0.01$ indicate statistically significant differences as compared with the normal liver. SOD, superoxide dismutase; GSS, glutathione synthetase; ROS, reactive oxygen species.
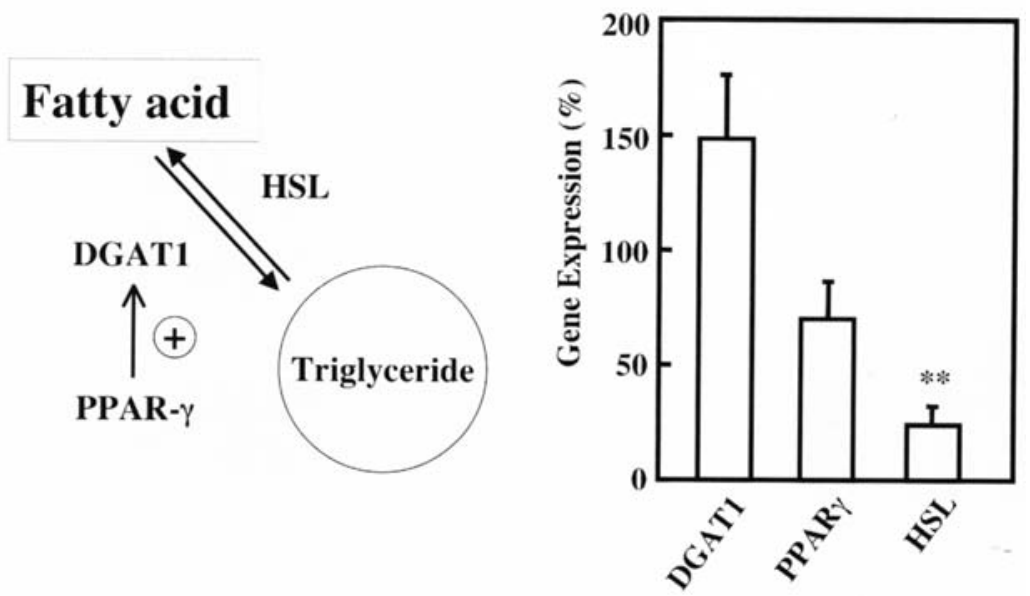

Figure 5. Real time RT-PCR analysis for expression of lipid droplet formation-related genes in NAFLD. ${ }^{* *}$ p $<0.001$ indicates statistically significant difference compared with the normal liver. DGAT1, diacylglycerol O-acyltransferase 1; PPAR $\gamma$, peroxisome proliferator-activated receptor $\gamma$; HSL, hormone-sensitive lipase.

triglycerides, which are stored within hepatocytes as lipid droplets. Diacylglycerol O-acyltransferase 1 (DGAT1) is involved in triglyceride synthesis, and its expression in NAFLD was increased 2-fold compared to that in the normal liver (Fig. 5). Expression of PPAR $\gamma$, which facilitates the storage of triglycerides in NAFLD, was 1.5 times greater than that of the controls. Hormone-sensitive lipase (HSL) is a key enzyme for catalyzing triglyceride accumulation in the form of lipid droplets. The expression of HSL was decreased by $80 \%$ in NAFLD as compared with the normal liver (Fig. 5).

\section{Discussion}

We previously reported a study on the expression of genes related to fatty acid metabolism in NAFLD (9). In the present study, we evaluated a wider range of genes and used a greater number of samples from both NAFLD and normal livers. Fatty acid metabolism in hepatocytes can occur by four mechanisms: a) de novo fatty acid synthesis and uptake of plasma-free fatty acids; b) fatty acid catalysis by oxidation in mitochondria, peroxisomes, and microsomes; c) neutral- ization of ROS derived from fatty acid oxidation; and d) conversion between fatty acids and triglycerides.

With respect to de novo fatty acid synthesis, the expression of ACC1 and FAS was increased in NAFLD, while expression of ACC2 was not. It has been reported that humans (20) and mice (21) with hepatic steatosis accumulate excess oleic acid (C18:1), the end-product of de novo fatty acid synthesis. This evidence, taken together with our results, suggests that fatty acid synthesis rates are increased in NAFLD despite the accumulation of fatty acids. In normal liver, fatty acid synthesis is positively regulated by transcriptional factor SREBP-1c, and in fatty acid overload, de novo fatty acid synthesis is suppressed through down-regulation of SREBP-1c (1). In NAFLD, the expression of SREBP-1c was increased 2 -fold, indicating that negative feedback regulation via SREBP-1c failed to occur.

In addition to an increase in de novo fatty acid synthesis, fatty acid uptake from serum can contribute to accumulation of fatty acids in NAFLD. ADRP has been suggested to play a role in fatty acid transport, although its function is not fully understood. It has been reported that ADRP-knockout mice 


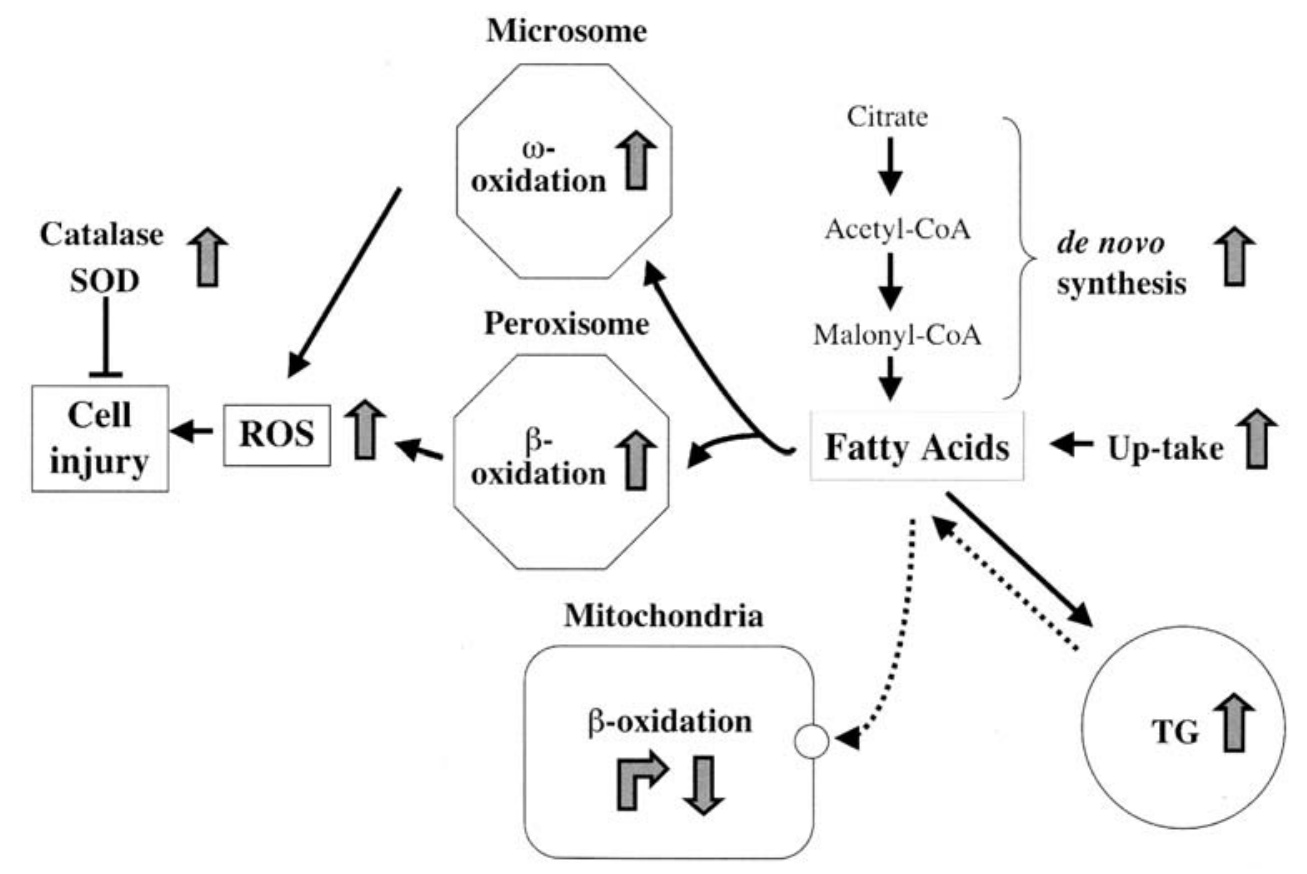

Figure 6. Summary of the present analysis of expression of fatty acid metabolism-related genes in NAFLD.

are resistant to the development of fatty liver (10). In NAFLD, the expression of ADRP was increased 2-fold and our findings are in agreement with the results of immunohistochemical studies in NAFLD by Motomura et al (13). It is very intriguing that not only de novo fatty acid synthesis was increased but also fatty acid uptake from serum, despite the excess hepatic accumulation of fatty acids in NAFLD.

Oxidation of fatty acids occurs mainly in the mitochondria. In this study, the expression of CPT1a was decreased, whereas that of LCAD, HADH $\alpha$, and UCP2 was increased. CPT1a is a membrane transporter of fatty acids (acyl-CoA) from the cytoplasm into the mitochondrial matrix and is a primary regulatory enzyme involved in mitochondrial $ß$ oxidation. The down-regulation of CTP1a expression in NAFLD is attributable to an increase in malonyl-CoA (14), since the expression of ACC1 which converts acetyl-CoA to malonyl-CoA, was increased (Fig. 1). Therefore, it is possible that $\beta$-oxidation in the mitochondria might be reduced in NAFLD. On the other hand, increased expression of LCAD and $\mathrm{HADH} \alpha$ suggests enhancement of $\beta$-oxidation. Furthermore, up-regulation of UCP2 expression suggests that excess ROS production occurs by increased $\beta$-oxidation because UCP2 potentially reduces ROS production $(15,16)$. When B-oxidation reaches maximal levels, a lack of unesterified CoA could inhibit CPT and thereby prevent further entry of acyl groups to the mitochondria, thus serving as an intramitochondrial control on ß-oxidation (17). Boxidation in mitochondria was at maximal levels, thereby resulting in decreased expression of CTP1a.

When there is an excess of fatty acids in hepatocytes, alternative pathways of fatty acid oxidation are activated, such as $\beta$-oxidation in peroxisomes and $\omega$-oxidation in the endoplasmic reticulum (microsomes). The peroxisomal acylCoA oxidases ACOX and BOX are the first and rate-limiting enzymes of $\beta$-oxidation pathways in peroxisomes (18), and their expression was increased in this study, indicating that peroxisomal $\beta$-oxidation is compensatively enhanced in NAFLD. In the endoplasmic reticulum, $\omega$-oxidation by CYP2E1 and CYP4As occurs (19). We demonstrated that the expression of both CYP2E1 and CYP4A11 was up-regulated in NAFLD. Increased activity of CYP2E1 in the liver is associated with factors commonly observed in NAFLD; obesity, diabetes, and hyperlipidemia. CYP4As are also assumed as key intermediaries in adaptive responses to the perturbations of hepatic lipid metabolism that accompany fasting, diabetes, and overnutrition. Recent evidence obtained from CYP2E1-null mice demonstrates that there is a compensatory increase in CYP4A activity (20). Therefore, in NAFLD, situations where CYP2E1 is fully activated may lead to increased CYP4A11 expression. On the whole, it appears that accumulation of fatty acids in NAFLD enhances oxidation not only in mitochondria but also in peroxisomes and microsomes.

PPAR $\alpha$ is a major transcriptional activator of genes involved in mitochondrial $\beta$-oxidation, such as peroxisomal ACOX and microsomal CYP4As $(18,21)$. In our study, the expression of PPAR $\alpha$ was unexpectedly reduced in NAFLD. When we compared the expression of ACOX to that of $\mathrm{BOX}$, which is not regulated by $\operatorname{PPAR} \alpha$, we found that the enhancement of expression of ACOX was less than that of BOX (2-fold vs. 10-fold, respectively) (Fig. 3). Similarly, CYP4A11 showed less enhancement of expression than did CYP2E1 (4-fold vs. 14-fold, respectively). The difference in the expression of oxidation enzymes in peroxisomes and microsomes might be attributable to the decreased expression of PPAR $\alpha$. We initially found that HOMA-IR, an index of insulin resistance, was negatively correlated with PPAR $\alpha$ expression, suggesting that the decreased expression of PPAR $\alpha$ may be attributable to insulin resistance, which often accompanies NAFLD. Recent studies have demonstrated that 
PPAR $\alpha$ agonists reduce hepatic steatosis in animal models $(22,23)$. Further study will be needed to clarify the mechanism of down-regulation of PPAR $\alpha$ and the effects of PPAR $\alpha$ activation as a treatment for NAFLD.

We investigated the expression of genes related to antioxidant pathways including SOD, catalase, and GSS, because it was expected that ROS overproduction would occur as a result of enhanced mitochondrial and peroxisomal ß-oxidation and microsomal $\omega$-oxidation, as described above. As we expected, the expression of SOD and catalase was dramatically enhanced. In contrast, the expression of GSS, which produces glutathione, was unchanged. Although we do not know precisely why GSS levels were unchanged, the antioxidant effects of glutathione can also be regulated by glutathione peroxidase, which, together with glutathione, neutralizes ROS.

Excess lipid droplet formation in NAFLD is indicative of increased triglyceride synthesis in hepatocytes, and in the present study, we observed increased expression of DGAT1. The expression of PPAR $\gamma$, which is a transcriptional factor that facilitates adipogenesis by various mechanisms including induction of DGAT1 expression, was unexpectedly unchanged in NAFLD. It has been reported that the expression of PPAR $\gamma$ is markedly increased in fatty liver (24). Conversely, adipogenesis resulting in triglyceride storage occurs under conditions where there is a decrease in PPAR $\alpha$ activity and fatty acid oxidation (21), implying that cross-talk occurs between PPAR $\gamma$ and PPAR $\alpha$. In this study, the expression of both PPAR $\gamma$ and PPAR $\alpha$ was decreased, suggesting that the cross-talk between these receptors might be impaired in NAFLD. Expression of HSL was also greatly down-regulated, indicating that lipolysis, in contrast to lipogenesis, is inhibited in NAFLD. Further study is needed to clarify the mechanisms that regulate expression of HSL and protein kinase A, which is a major regulator of HSL expression (25).

In summary, our results in patients with NAFLD indicate that: i) de novo synthesis of fatty acids is increased, although fatty acids have already been accumulated in hepatocytes, and is accompanied by increased fatty acid uptake from serum; ii) mitochondrial fatty acid oxidation is decreased or fully activated to improve fatty acid accumulation; iii) in order to complement the function of mitochondria ( $(-$ oxidation), peroxisomal ( $\beta$-oxidation) and microsomal $(\omega-$ oxidation) oxidation is up-regulated; iv) antioxidant pathways including SOD and catalase are enhanced to neutralize overproduced ROS by enhanced oxidation; and v) lipid droplet formation is enhanced (Fig. 6). Four cases of histologically proven NASH were included in the present study, and the gene expression profiles did not differ between patients with NASH and those with NAFLD (data not shown). Since ROS are believed to be a major cytotoxic factor in NASH $(19,26,27)$, it is assumed that uncompensated ROS overproduction due to enhanced oxidation might lead to the transition from simple obesity to NASH, a condition in which excessive ROS production can cause mitochondrial failure leading to apoptosis and oncosis (necrapoptosis) (28-30).

Eleven of the NAFLD patients in this study, who were candidates as donors for liver transplantation received a strict low-calorie diet, exercise, and $400 \mathrm{mg}$ /day bezafibrate (a ligand of PPAR $\alpha$ ) for 4-8 weeks prior to the operations (31). We found that this treatment normalized dysfunctional expression of genes related to fatty acid metabolism, i.e. ACC1 and FAS expression were decreased and CTP1a and PPAR $\alpha$ expression were increased (data not shown). Therefore, treatments that target the expression of fatty acid metabolismrelated genes may be beneficial in NAFLD.

Finally, as discussed above, several disorders of fatty acid metabolism were recognized in NAFLD, and we assume that unregulated enhancement of de novo fatty acid synthesis is a primary disorder in NAFLD. Fatty acid synthesis by ACC1 and FAS is tightly regulated by SREBP-1c and its expression is also regulated negatively by AMP-activated protein kinase (AMPK) and positively by insulin $(1,32)$. Obesity, which is often accompanied by NAFLD, causes decreasing serum levels of adiponectin and increasing levels of $\mathrm{TNF} \alpha$. It has been reported that decreased adiponectin and/or increased TNG $\alpha$ activity results in decreased SREBP-1c expression $(33,34)$. Insulin resistance, a condition in which insulinsignaling pathways are suppressed, was also commonly observed in NAFLD which presumably caused a decrease in SREBP-1c expression. We are now investigating AMPK expression and insulin-receptor substrates which are key molecules in the insulin signaling cascade affecting lipid metabolism (35).

\section{References}

1. Browning JD and Horton JD: Molecular mediators of hepatic steatosis and liver injury. J Clin Invest 114: 147-152, 2004.

2. Clark JM, Brancati FL and Diehl AM: The prevalence and etiology of elevated aminotransferase levels in the United States. Am J Gastroenterol 98: 960-967, 2003.

3. Angulo P: Nonalcoholic fatty liver disease. N Engl J Med 346: 1221-1231, 2002.

4. Nomura H, Kashiwagi S, Hayashi J, Kajiyama W, Tani S and Goto M: Prevalence of fatty liver in a general population in Okinawa, Japan. Jpn J Med 27: 142-149, 1988.

5. Hilden M, Christoffersen P, Juhl E and Dalgaard JB: Liver histology in a 'normal' population - examination of 503 consecutive fatal traffic casualties. Scand J Gastroenterol 12: 593-597, 1977.

6. Bellentani S, Saccoccio G, Masutti F, et al: Prevalence of and risk factors for hepatic steatosis in northern Italy. Ann Intern Med 132: 112-117, 2000.

7. Ludwig J, Viggiano TR, McGill DB and Oh BJ: Nonalcoholic steatohepatitis: Mayo Clinic experiences with a hitherto unnamed disease. Mayo Clin Proc 55: 434-438, 1980.

8. Matteoni CA, Younossi ZM, Gramlich T, Boparai N, Liu YC and McCullough AJ: Nonalcoholic fatty liver disease: a spectrum of clinical and pathological severity. Gastroenterology 116: 1413-1419, 1999.

9. Nakamuta M, Kohjima M, Morizono S, Kotoh K, Yoshimoto T, Miyagi I and Enjoji M: Evaluation of fatty acid metabolismrelated gene expression in nonalcoholic fatty liver disease. Int $\mathbf{J}$ Mol Med 16: 631-635, 2005.

10. Chang BH, Li L, Paul A, Taniguchi S, Nannegari V, Heird WC and Chan L: Protection against fatty liver but normal adipogenesis in mice lacking adipose differentiation-related protein. Mol Cell Biol 26: 1063-1076, 2006.

11. Negre-Salvayre A, Hirtz C, Carrera G, et al: A role for uncoupling protein-2 as a regulator of mitochondrial hydrogen peroxide generation. FASEB J 11: 809-815, 1997.

12. Krauss S, Zhang CY and Lowell BB: A significant portion of mitochondrial proton leak in intact thymocytes depends on expression of UCP2. Proc Natl Acad Sci USA 99: 118-122, 2002.

13. Motomura W, Inoue M, Ohtake T, et al: Up-regulation of ADRP in fatty liver in human and liver steatosis in mice fed with high fat diet. Biochem Biophys Res Commun 340: 1111-1118, 2006. 
14. McGarry JD, Mannaerts GP and Foster DW: A possible role for malonyl-CoA in the regulation of hepatic fatty acid oxidation and ketogenesis. J Clin Invest 60: 265-270, 1977.

15. Pessayre D, Berson A, Fromenty B and Mansouri A: Mitochondria in steatohepatitis. Semin Liver Dis 21: 57-69, 2001.

16. Skulachev VP: Uncoupling: new approaches to an old problem of bioenergetics. Biochim Biophys Acta 1363: 100-124, 1998.

17. Bartlett $\mathrm{K}$ and Eaton S: Mitochondrial beta-oxidation. Eur J Biochem 271: 462-469, 2004.

18. Reddy JK and Hashimoto T: Peroxisomal beta-oxidation and peroxisome proliferator-activated receptor alpha: an adaptive metabolic system. Annu Rev Nutr 21: 193-230, 2001.

19. Robertson G, Leclercq I and Farrell GC: Nonalcoholic steatosis and steatohepatitis. II. Cytochrome P-450 enzymes and oxidative stress. Am J Physiol Gastrointest Liver Physiol 281: G1135-G1139, 2001.

20. Enriquez A, Leclercq I, Farrell GC and Robertson G: Altered expression of hepatic CYP2E1 and CYP4A in obese, diabetic ob/ob mice, and fa/fa Zucker rats. Biochem Biophys Res Commun 255: 300-306, 1999.

21. Reddy JK: Nonalcoholic steatosis and steatohepatitis. III. Peroxisomal beta-oxidation, PPAR alpha, and steatohepatitis. Am J Physiol Gastrointest Liver Physiol 281: G1333-G1339, 2001.

22. Ip E, Farrell G, Hall P, Robertson G and Leclercq I: Administration of the potent PPARalpha agonist, Wy-14,643, reverses nutritional fibrosis and steatohepatitis in mice. Hepatology 39: 1286-1296, 2004.

23. Harano Y, Yasui K, Toyama T, et al: Fenofibrate, a peroxisome proliferator-activated receptor alpha agonist, reduces hepatic steatosis and lipid peroxidation in fatty liver Shionogi mice with hereditary fatty liver. Liver Int 26: 613-620, 2006.

24. Chao L, Marcus-Samuels B, Mason MM, et al: Adipose tissue is required for the antidiabetic, but not for the hypolipidemic, effect of thiazolidinediones. J Clin Invest 106: 1221-1228, 2000 .
25. Holm C: Molecular mechanisms regulating hormone-sensitive lipase and lipolysis. Biochem Soc Trans 31: 1120-1124, 2003.

26. Seki S, Kitada T, Yamada T, Sakaguchi H, Nakatani K and Wakasa K: In situ detection of lipid peroxidation and oxidative DNA damage in non-alcoholic fatty liver diseases. J Hepatol 37 : 56-62, 2002.

27. Bergamini CM, Gambetti S, Dondi A and Cervellati C: Oxygen, reactive oxygen species and tissue damage. Curr Pharm Des 10: 1611-1626, 2004

28. Perez-Carreras M, Del Hoyo P, Martin MA, et al: Defective hepatic mitochondrial respiratory chain in patients with nonalcoholic steatohepatitis. Hepatology 38: 999-1007, 2003.

29. Fromenty B, Robin MA, Igoudjil A, Mansouri A and Pessayre D: The ins and outs of mitochondrial dysfunction in NASH. Diabetes Metab 30: 121-138, 2004.

30. Jaeschke $\mathrm{H}$ and Lemasters JJ: Apoptosis versus oncotic necrosis in hepatic ischemia/reperfusion injury. Gastroenterology 125: 1246-1457, 2003.

31. Nakamuta M, Morizono S, Soejima Y, et al: Short-term intensive treatment for donors with hepatic steatosis in living-donor liver transplantation. Transplantation 80: 608-612, 2005.

32. Shimomura I, Bashmakov Y and Horton JD: Increased levels of nuclear SREBP-1c associated with fatty livers in two mouse models of diabetes mellitus. J Biol Chem 274: 30028-30032, 1999.

33. Viollet B, Foretz M, Guigas B, et al: Activation of AMP-activated protein kinase in the liver: a new strategy for the management of metabolic hepatic disorders. J Physiol 574: 41-53, 2006.

34. Whitehead JP, Richards AA, Hickman IJ, Macdonald GA and Prins JB: Adiponectin - a key adipokine in the metabolic syndrome. Diabetes Obes Metab 8: 264-280, 2006.

35. Taniguchi CM, Ueki K and Kahn R. Complementary roles of IRS-1 and IRS-2 in the hepatic regulation of metabolism. J Clin Invest 115: 718-727, 2005. 